\title{
Evaluation and Characterization of Orbital Lesions using 128 Slice Multidetector Computer Tomography
}

\author{
Vijaykumar K. R. ${ }^{1}$, Ramanjaneyalu. $\mathbf{N}^{2}$, Lohith Yadav ${ }^{3}$ \\ ${ }^{1}$ Professor, Department of Radiodiagnosis, Bangalore Medical College and Research Institute, ${ }^{2}$ Postgraduate Resident, \\ Department of Radiodiagnosis, Bangalore medical college and Research Institute, ${ }^{3}$ Postgraduate Resident, Department of \\ Radiodiagnosis, Bangalore Medical College and Research Institute, India
}

Corresponding author: Dr. Ramanjaneyalu. N, No.002, Koncept Nakshatra Apartment, Nagarbhavi, Bengaluru - 560072, India

DOI: http://dx.doi.org/10.21276/ijcmsr.2020.5.2.25

How to cite this article: Vijaykumar. K. R., Ramanjaneyalu. N, Lohith Yadav. Evaluation and characterization of orbital lesions using 128 slice multidetector computer tomography. International Journal of Contemporary Medicine Surgery and Radiology. 2020;5(2):B101-B105.

\section{A B S T R A C T}

Introduction: The sophisticated and complex nature of orbital anatomy, presence of tiny and vital structures in relationship with surrounding facial bones make the diagnosis of orbital diseases extremely challenging. Often misdiagnosis and incomplete treatment of orbital disorders leads to recurrence, functional and cosmetic sequalae. Study aimed to evaluate 128 slice multi detector computer tomography as a modality to identify various orbital lesions and to characterize and document various orbital lesions on 128 slice MDCT with histopathological Correlation.

Method and Materials: It was a prospective study conducted in our institute from July 2017 to December 2018 with a sample size of 50.50 patients were evaluated in this study conducted at Dept of Radio-diagnosis, Bangalore medical college and research institute. All patients were subjected to orbital CT scans on a fourth generation 128 slice multidetector CT scanner (Philips-Ingenuity Core 128).

Results: Thyroid ophthalmopathy was seen in $20 \%$ of the study subjects, followed by orbital cellulitis (12\%), left chronic dacrocystitis (8\%), left orbital malignancy (3\%), dermoid (3\%), bilateral lacrimal adenitis (3\%). Out of 50 (100\%) subjects, more than half of the subjects were males- 28 (56\%) whereas 22 (44\%) were females in the present study. Among the study subjects, majority of them belonged to the age group of 0-9 years (16\%), followed by 20-29, 30-39, 40-49, 50-59, 70-79 yrs each comprising of $6(12 \%)$ subjects).

Conclusion: 128 Multidetector MDCT offers unique opportunity for evaluation of pathologies in multiple planes, can generate images in any plane without anatomical distortion. MDCT is effective tool in arriving at specific diagnosis. Very effective tool in identifying the exact anatomic site of lesion, thus helps in arriving at a diagnosis and their by helps in further planning of treatment.

Keywords: MDCT, Multidetector Computer Tomography

\section{INTRODUCTION}

The sophisticated and complex nature of orbital anatomy, presence of tiny and vital structures in relationship with surrounding facial bones make the diagnosis of orbital diseases extremely challenging. Often misdiagnosis and incomplete treatment of orbital disorders leads to recurrence, functional and cosmetic sequalae. ${ }^{1}$

Radiograph play an important role but has diagnostic limitation in acute craniofacial trauma (CFT) cases due to superimposition of surrounding bony structures, impaired visualization of underlying fine fractures due to periorbital oedema, ecchymosis and haemorrhage. ${ }^{1}$

Findings on plain radiographs \& ultrasonography are not pathognomic of most of the orbital disease process though some help can be obtained in characterization of lesion in certain cases. Advent of CT\& MRI has revolutionized the diagnostic imaging of orbit $\&$ its contents. ${ }^{2}$

Advent of CT\& MRI has revolutionized the diagnostic imaging of orbit \& its contents. MRI with its superb soft tissue contrast \&multiplanar ability provides excellent rendering of orbital anatomy but is limited by lack of wider availability $\&$ high cost. $^{2}$ On the other hand easy availability and operability, good maintenance and speed makes CT scan an affordable diagnostic tool in orbital diseases under existing circumstances and present set up. ${ }^{2}$

Computerized tomography $(\mathrm{CT})$ is a simple, safe and noninvasive diagnostic tool performed on an OPD basis. The information through $\mathrm{CT}$ scan can be enhanced by several special techniques like contrast enhancement, narrow density, window measurement, three dimensional $\mathrm{CT}$ and image reversal. ${ }^{1}$

The basic anatomical unit of vision, ocular apparatus is housed in a four sided pyramid - orbit. The anatomical relation of the orbit to paranasal sinuses make it susceptible to pathologies of the adjacent sinuses apart from intraorbital pathologies. CT provides us excellent delineation of bony orbit and the adjoining soft tissues. ${ }^{3}$ 
Orbital injuries are often seen in persons with polytrauma/ major trauma after road traffic accident, physical assault, fall from height and industrial or domestic accidents.

These orbital injuries are often severe and complex in nature involving skull and face.

Appropriate management of acute orbital and craniofacial trauma (CFT) requires intense and immediate clinical decision, which is dependent on radiological depiction of injury $^{1}$

Orbital mass lesions include wide spectrum of pathology ranging from orbital inflammatory lesions, cysts, vascular anomalies and metastasis to tumors (benign and malignant). These lesions are challenging problems frequently faced by radiologists and ophthalmologists. ${ }^{3}$

The most common lesion causing proptosis were tumors. Bilateral eye involvement with extraocular muscle involvement is more common with graves disease.

In inflammatory pseudotumor of the orbit, unilateral involvement was more common than bilateral. mucoceles of paranasal sinuses invading the orbit, involvement of frontal sinus was most common followed by fronto-ethmoid involvement. $^{4}$

Orbital neoplasms in adults comprise a broad spectrum of benign and malignant entities. Cross-sectional imaging plays a valuable role in characterization of these lesions and in evaluation of disease extent, supplementing clinical ophthalmologic examination and providing information beyond what can be seen at fundoscopy. ${ }^{5}$

Several approaches to the diagnosis of orbital pathology are in use. A common strategy is to localize the pathology to one of the defined compartments of the orbit. ${ }^{5}$ Disease processes can be classified according to the anatomical site involved this is usually in relation to the rectus muscle pyramid (the "cone")

1. Intraconal (within the muscle cone)

2. Arising from the muscle cone

3. Extraconal (outside the muscle cone)

4. Within or involving the globe. ${ }^{5}$

Most diffuse processes involving the orbit are inflammatory or infectious in origin. Patients often present with restriction of motion of the globe, proptosis and pain. Systemic symptoms and signs may be present. Imaging is performed to define the aetiology and extent of disease, and to assess the response to therapy. ${ }^{6}$

Ushalatha et.al conducted a study on 50 patients referred for orbital mass and found that orbital pseudo tumor(26\%) was most common followed by lymphoma(18\%).

In pediatric age group they found that Optic nerve Glioma(10\%) was the common lesion followed by hemangioma (8\%) and dermoid cyst (6\%). Accuracy of Computed Tomography in the diagnosis of orbital tumors was $86 \%$ with more sensitivity for diagnosing benign lesions (90.3\%) than malignant lesions (78.9\%). ${ }^{7}$

Study aimed to evaluate 128 slice multi detector computer tomography as a modality to identify various orbital lesions and to characterize and document various orbital lesions on 128 slice MDCT with histopathological Correlation.

\section{MATERIAL AND METHODS}

The study was conducted in Department of Radiology, Bangalore medical college and research institute, Bengaluru. Patients clinically suspected of having trauma to the orbits, proptosis, pain, visual impairment with or without ophthalmoplegia, orbital mass were subjected ophthalmological examination and CT orbit. CT findings were correlated with final diagnosis based on clinical, laboratory, operative findings, histopathological study or response to treatment.

\section{Inclusion criteria}

- All patients clinically suspected to have orbital lesions referred to the department of Radio diagnosis in a period of one and a half years from November 2017 to May 2018 will be subjected for the study.

- Any lesion originating in the orbit.

- Any lesion invading the orbit from paranasal sinuses, nose or brain.

- Cases of all age groups

\section{Exclusion criteria}

- Patients with pre existing orbital fractures

- Post operative and patients undergoing chemotherapy and radiotherapy

- Patients in whom contrast administration is contraindicated.

\section{Methodology}

All patients included in the study were explained of the procedure and informed written consent will be taken from them.

A structured pre-prepared case proforma was used to enter the patient details, clinical history, physical examination.

Patients were subjected to Computed Tomography examination prior to and after administration of Intravenous contrast medium 128 MDCT9(PHILIPS). Protocol used

- Contiguous axial and coronal sections with $1 \mathrm{~mm}$ thin sections slice thickness.

The lesions were assessed according to the CT findings:

Location - Extraconal/Intraconal/Both/involving

lacrimal fossa.

Attenuation - Isodense/Hyperdense/Hypodense/

Heterogenous.

Muscle involvement - Present/Absent.

Bony changes - Absent/Remodelling/Destruction/

Infiltration.

Calcification - Present/Absent.

Optic nerve involvement - Present/Absent.

Paranasal sinuses involvement - Present/Absent.

Enhancement - Present/Absent/Rim.

and other characteristics.

Computed Tomographic findings and diagnosis will then be correlated with US/MRI wherever applicable.

\section{STATISTICAL ANALYSIS}

Statistical analyses were made with IBM SPSS software version 20.0 and Chi-Square test was applied to determine the statistical significance. 


\section{RESULTS}

Out of $50(100 \%)$ subjects, more than half of the subjects were males- 28(56\%) whereas $22(44 \%)$ were females in the present study (table-1).

Among the study subjects, majority of them belonged to the age group of 0-9 years (16\%), followed by 20-29, 30-39, 40$49,50-59,70-79$ yrs each comprising of 6 (12\%) subjects) (table-2).

Out of 50 (100\%) subjects, Left side eye was more involved-21

\begin{tabular}{|l|c|c|}
\hline Gender & Frequency & Percent \\
\hline Females & 22 & 44.0 \\
\hline Males & 28 & 56.0 \\
\hline Total & 50 & 100.0 \\
\hline \multicolumn{2}{|c|}{ Table-1: Distribution of the subjects based on gender } \\
\hline
\end{tabular}

\begin{tabular}{|l|c|c|}
\hline Age groups & Frequency & Percent \\
\hline 0 to 9 yrs & 8 & 16.0 \\
\hline 10 to 19 yrs & 5 & 10.0 \\
\hline 20 to 29 yrs & 6 & 12.0 \\
\hline 30 to 39 yrs & 6 & 12.0 \\
\hline 40 to 49 yrs & 6 & 12.0 \\
\hline 50 to 59 yrs & 6 & 12.0 \\
\hline 60 to 69 yrs & 5 & 10.0 \\
\hline 70 to 79 yrs & 6 & 12.0 \\
\hline Above 80 yrs & 2 & 4.0 \\
\hline Total & 50 & 100.0 \\
\hline \multicolumn{2}{|c|}{ Table-2: Distribution of the subjects based on age } \\
\hline
\end{tabular}

\begin{tabular}{|l|c|c|}
\hline Eye involvement & Frequency & Percent \\
\hline Bilateral & 15 & 30.0 \\
\hline Left & 21 & 42.0 \\
\hline Right & 14 & 28.0 \\
\hline Total & 50 & 100.0 \\
\hline \multicolumn{2}{|c|}{ Table-3: Distribution of the subjects based on eye } \\
involvement \\
\hline
\end{tabular}

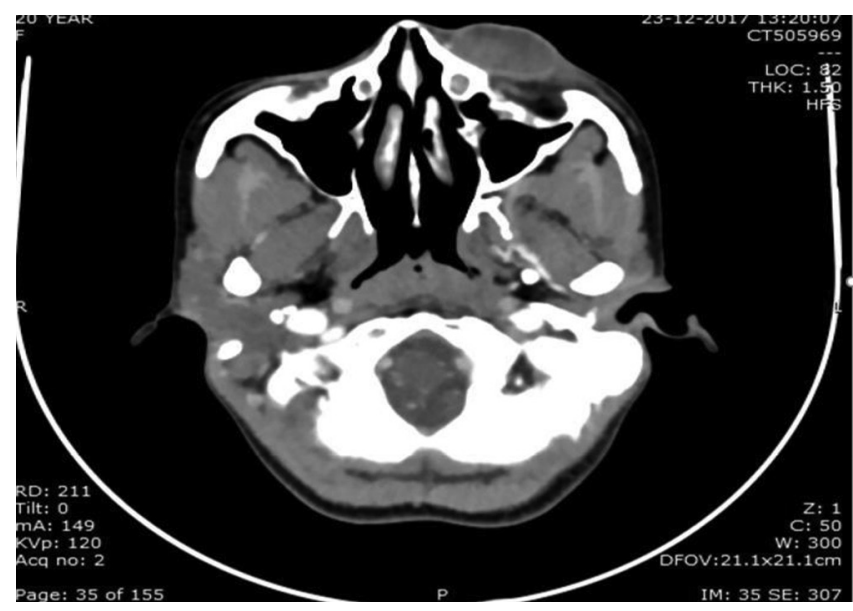

Image-1: A case of chronic dacrocystitis.

Well defined cystic lesion showing peripheral enhacement with central enhacing area and fluid attenuating non enhancing area within in the left lower eye lid extending to the lacrimal sac and causing widening of nasolacrimal duct.
(42\%) followed by $15(30 \%)$

bilateral involvement and 14 (28\%) had right eye involvement (table-3).

Thyroid ophthalmopathy was seen in $20 \%$ of the study subjects, followed by orbital cellulitis (12\%), left chronic

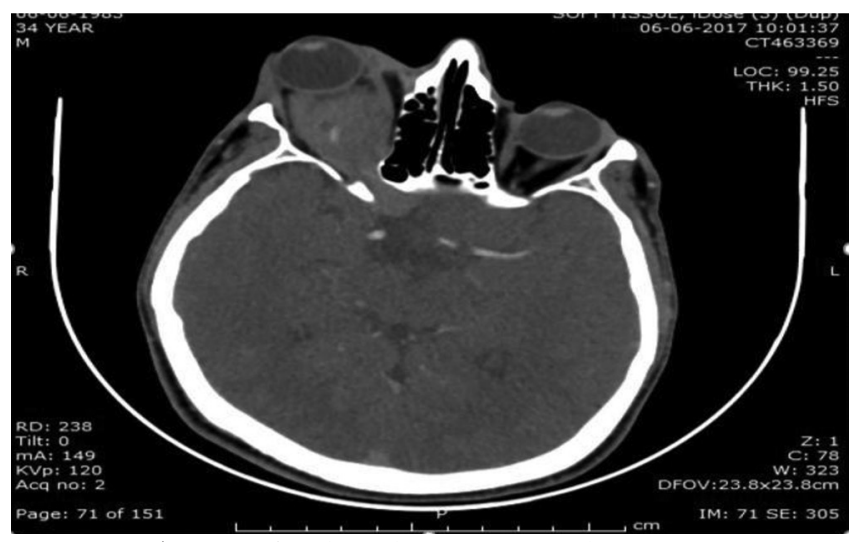

Image-2: A case of right orbit cavernous hemangioma

Well defined intra-conal soft tissue attenuating lesion right orbit. The lesion seen encasing the optic nerve, and opthalmic artery. Intracranial extension of the lesion noted with mild homogeneous enhancement on post contrast study.

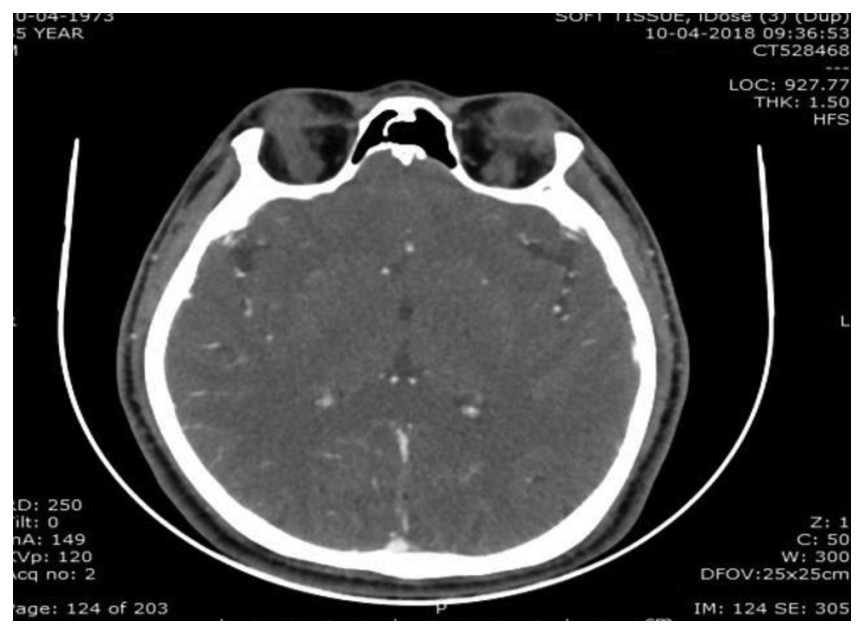

Image-3: A case of thyroid ophthalmopathy.

Axial CECT shows bulky bilateral inferior recti muscles.

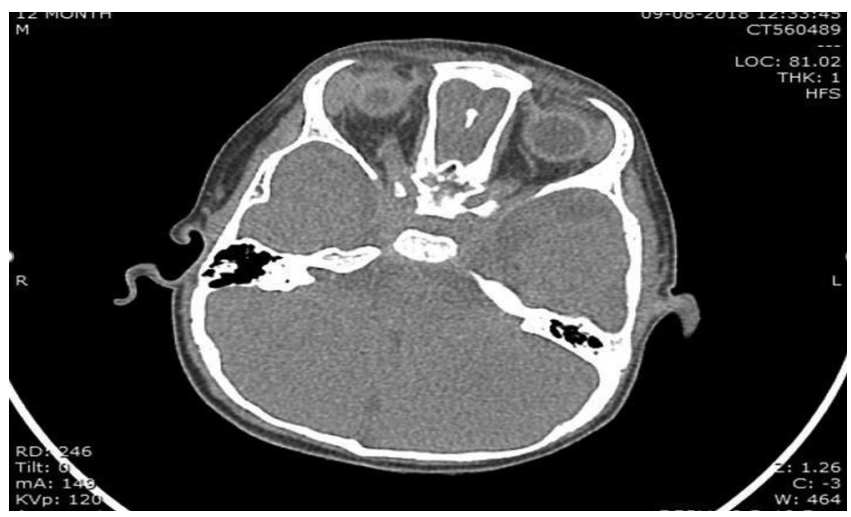

Image-4: A case of bilateral lacrimal adenitis.

Axial CT shows bilateral pre and post septal thickening. Bilateral lacrimal glands are bulky in size with surrounding fat stranding. 


\begin{tabular}{|l|c|c|}
\hline & Frequency & Percent \\
\hline Bilateral lacrimal adenitis & 3 & 6.0 \\
\hline Capillary hemangioma & 1 & 2.0 \\
\hline $\begin{array}{l}\text { Left upper eye lid squamous cell } \\
\text { carcinoma }\end{array}$ & 1 & 2.0 \\
\hline Cavernous hemangioma & 1 & 2.0 \\
\hline Dermoid & 3 & 6.0 \\
\hline Epidermoid & 1 & 2.0 \\
\hline Fatty atrophy of left lateral rectus & 1 & 2.0 \\
\hline Fibroma of left lacrimal sac & 1 & 2.0 \\
\hline left acute dacrocystitis & 1 & 2.0 \\
\hline left chronic dacrocystitis & 4 & 8.0 \\
\hline Left orbital malignacy & 3 & 6.0 \\
\hline Low flow venous malformation & 1 & 2.0 \\
\hline Maxillary sinus carcinoma & 1 & 2.0 \\
\hline Mucocele of left frontal sinus & 2 & 4.0 \\
\hline Neurofibroma & 1 & 2.0 \\
\hline Orbital celluitis & 6 & 12.0 \\
\hline Orbital lymphoma & 2 & 4.0 \\
\hline Orbital pseudotumor & 2 & 4.0 \\
\hline Right lacrimal gland adenitis & 1 & 2.0 \\
\hline Right lacrimal gland adenocarcinoma & 1 & 2.0 \\
\hline Right medial canthus dermoid & 1 & 2.0 \\
\hline Right sinonasal carcinoma & 1 & 2.0 \\
\hline Right upper lid Squamous cell carci- \\
noma & 1 & 2.0 \\
\hline Thryoid ophthalmopaty & 10 & 20.0 \\
\hline Total & 50 & 100.0 \\
\hline Table-4: Distribution of the subjects based on CT changes \\
\hline
\end{tabular}

dacrocystitis (8\%), left orbital malignancy (3\%), dermoid (3\%), bilateral lacrimal adenitis (3\%) (table-4).

\section{DISCUSSION}

Fifty patients with orbital lesions were evaluated. Age of patients ranged from 1 year to 80 yearrs $A$ slight male predominance was noted. Proptosis was most common complaint of patients in my study. My study is comparable upto some extent to previous studies. In pediatric age group dermoid is the most frequently occurring case In adults Graves disease and pseudotumor was more common. Orbital cellulitis was also more common lesion in my study. There were 35 unilateral cases and 15 bilateral cases. Extension outside orbit was seen in 13 cases. Orbital involvement from para orbital structures were seen in 27 cases. Bony changes were seen in 3 cases,

Vascular tumours: There were 1 cases of cavernous haemangioma. The lesion was hyperdense intraconal lesion and enhancing with contrast. There was no e/o calcification. No e/o any bony involvement. There was 1 case of slow flow venolymphatic malformation which was ill defined. Intraconal lesion which showed more prominent and enhanced lesion with contrast. There was 1 case of capillary haemangioma which was hyperdense lesion enhancing with contrast.

At CT, venous lymphatic malformations typically are poorly circumscribed, multicompartmental, and heterogeneously hyperattenuating, and they enhance minimally after contrast material administration. Phleboliths may be present ${ }^{8}$

Pseudotumor: There were 2 cases of pseudotumor 2 cases with bilateral involvement. In both the cases multiple muscle involvement was seen. In both the cases tendon was involved. Pseudotumor (idiopathic orbital inflammation) is the most common inflammatory lesion of the orbit. It usually occurs in the middle age and is characterized by the classic triad of proptosis, pain and impaired ocular motility. ${ }^{9}$

Thyroid Orbitopathy: 10 cases were seen. All 10 cases had B/L involvement. All 10 cases had multiple muscle involvement. All cases were enhancing with contrast. In all cases tendinous insertion spared.

Orbital cellulitis: 6 cases of orbital cellulitis were seen. Among all 3 were involving right and 3 were in left. All cases showed involvement of both pre and post septal regions.

Dermoid: 4 cases seen. Most of the cases were seen in paediatric population. All cases were hypodense lesions. No enhancement was seen in all the cases. Post operative HPE confirmed diagnosis all the case.

Mucocele: 2 cases of of frontal sinus mucocele seen. One was involving right and other was involving left. Intra cranial extension was seen in one case. There was bony erosion seen

Maxillary sinus Malignancy and sinonasal malignancy: 2 cases were seen Lesion were extending from maxillary sinus into orbit. CT diagnosis of locally infiltrative malignancy was made. Histopathology report of maxillary sinus malignancy was made.

Dacrocystitis: Nine case of dacdrocystitis were seen. Bilateral involvement seen in 3 cases. Two cases were acute dacrocystitis one was involving right and other was left.

Orbital lymphoma: Two cases of orbital lymphoma were seen. Bilateral involvement seen both cases. Both the lesions were ill defined. Diffuse involvement seen in both the cases.

Upper lid squamous carcinoma: Squamous cell carcinoma of upper lid involving right upper eye lid seen in 2 cases. and involving left eye lid seen in one case. Lesions showed intense enhancement on post contrast study. Diagnosis was confirmed by MRI.

Lacrimal gland adenocarcinoma: Right eye was involved in 25 year male patient Right lacrimal gland was bulky in size. Lesion showed heterogenous enhancement

Diagnosis was confirmed by MRI

Neurofibroma: 30 years female patient presented with swelling and edema of right eye. On CT examination there was Well defined extraconal soft tissue attenuating lesion. Multiple well defined hypodense lesions seen in bilateral parapharangeal spaces, posterior cervical space, subcutaneous plane of neck. HPE features were suggestive of neurofibroma.

Fibroma of left nasolacrimal sac: 17 years female presented with pain, edema and swelling of left eye. CT examination showed small well defined hyperdense nodule noted in medial canthus in the region. HPE confirmed diagnosis. 
Fatty atrophy of left lateral rectus: 27 years male presented with left sided visual impairment. CT examination: Axis of the left orbit is altered with the intraocular lens facing medially.

In a study conducted by E.A. Parthasarathy et all Among 102 patients trauma constituted the most common etiology, followed by inflammatory and neoplastic. Lesions were analyzed for calcification, bony and paranasal sinus involvement, enhancement characteristics, lacrimal gland and optic nerve involvement. ${ }^{10}$

Ben simon etal studied 131 biops proven cases of orbitallesions and found that Benign lesions were more likely to be smaller in size, round or oval in shape (29\% of all benign tumors), and associated with hyperostosis (22\% of all benign lesions). Inflammatory processes showed pan orbital involvement. Orbital fat involvement and fat stranding were noticed only in inflammatory lesions (19\% and 16\%, respectively). Malignant features such as pan orbital involvement, orbital fat, frontal sinus opacity, moulding around orbital structures, perineural involvement, and fat stranding had specificity of $97 \%$ to $100 \%$, but low sensitivity. ${ }^{11}$

\section{CONCLUSION}

CT is modality of choice for assessing orbital lesions. Better information obtained by appropriated scanning technique and systematic. analysis of CT characters of various orbital lesions. Appropriate window setting need to be done. Valsalva maneuver technique has to be used whenever needed. Axial, coronal and sagittal sections to the taken in order to improve diagnostic accuracy. Distribution of lesion differs in children and adults dermoid was most

common tumor in children followed by orbital cellulitis. Any extra-orbital extension or intracranial extension helps in assessing prognosis. Compartmentalization helps in arriving at a diagnosis. CT also helps monitoring response to treatment. CT was accurate in narrowing differential diagnosis.

\section{REFERENCES}

1. Maurya RP, Singh VP, Singh MK, Shrinkhal, Sen PR, Verma A, et al. Diagnostic value of three dimensional CT reconstruction in various orbital disorder. International Journal of Ocular Oncology and Oculoplasty, JanuaryMarch, 2016;2(1):48-60.

2. PK Shah, RK Rauniyar, MK Gupta, BP Badhu. Role of imaging (MDCT) in ocular and orbital lesions. Health Renaissance 2015;13(3): 24-36.

3. Dubey R B, Tara N P, Sisodiya K N. Computerised tomographic evaluation of orbital lesions: Pictorial essay.Indian J Radiol Imaging 2003;13(1):261-270

4. Sabharwal KK, Chouhan AL, Jain S. CT Evaluation of Proptosis. Ind J Radiollmag 2006; 16(4):683-688

5. Tailor TD, Gupta D, Dalley RW, Keene CD, Anzai Y. Orbital Neoplasms in adults: Clinical, Radiologic, and Pathologic Review.RadioGraphics 2013;33(2):1739_ 1758

6. Adam A, Dixon AK, Grainger RG, Allison DJ(eds): Grainger and Allison's diagnostic radiology essentials, 6th ed.Edinburgh: Churchill, Livingstone, 2013:820835
7. Ushalatha B,Sambasivarao K. Role of CT in the evaluation of orbital tumors. IOSR Journal of Dental and Medical Sciences 2016; 15(4):16-10.

8. Wendy R. K. Smoker, Lindell R. Gentry, Norbert K. Yee, Deborah L. Reede, Jeffrey A. Nerad. Vascular Lesions of the Orbit: More than Meets the Eye. Radio Graphics 2008; 28(1):185-204

9. Weber AL, Romo LV.Pseudotumour and orbit: Clinical pathological and radiological evaluation. Radiological Clinics of North America 1999; 37 (1): 151-168.

10. E.A. Parthasarathy, Einstien A, Abubacker Sulaiman F, Alex Daniel Prabhu, Ashraf Ahmed B, Saravanan K. Computed Tomography in Orbital Pathology with Clinicopathological Correlation. International Journal of Contemporary Medicine Surgery and Radiology. 2018;3(2):B60-B64

11. Simon BGJ, Annunziata CC, Fink J, Villablanca P, McCann JD, Goldberg RA. Rethinking orbital imaging - Establishing guidelines for interpreting orbital imaging studies and evaluating their predictive value in patients with orbital tumors. Ophthalmology 2005;112(12):2196-207.

Source of Support: Nil; Conflict of Interest: None

Submitted: 14-03-2020; Accepted: 17-05-2020; Published online: 26-06-2020 